\title{
Is precision medicine the future of healthcare?
}

Richard W Barker*,1

${ }^{1}$ Oxford-UCL Centre for the Advancement of Sustainable Medical Innovation (CASMI), University of Oxford, Oxford, UK

* Author for correspondence: richard.barker@casmi.org.uk

\author{
"Yes, precision medicine is the future of healthcare, but its arrival is very unevenly distributed."
}

First draft submitted: 7 August 2017; Accepted for publication: 17 August 2017; Published online: 24 November 2017

Keywords: genomics • personalized medicine • precision medicine • stratified medicine

"The future is already here, it's just not very evenly distributed" said William Gibson about the cybersphere. I will argue similarly of precision medicine (PM). Yes, precision medicine is the future of healthcare, but its arrival is very unevenly distributed.

As we address our question let us ask: how should we define 'precision medicine'; how will science shape it; what nonscientific forces might drive or impede its progress; where will we see it first; and which stakeholders need to act to make it a reality?

\section{PM: a broad definition for a new era of healthcare}

There are heated debates about semantics: should we talk about stratified medicine, personalized medicine or individualized medicine? The value of the term 'precision' is that it can embrace the whole spectrum, from prompting us to segment large populations into subgroups requiring different therapy, to exquisitely personalizing treatment. What is in common across this spectrum is the use of objective measures to characterize the patient and their disease at a molecular level and thus guide the selection of treatment.

But what measures or markers to focus on? Some define PM quite narrowly, focusing on the power of genomics to determine the right therapy for an individual. We already see this in clinical practice, of course, in the treatment of many cancers and rare diseases, at least in most developed economies.

However, this genomic definition is too narrow, as we know that many major diseases are multigenic and are influenced by many phenotypic factors, from lifestyle choices (shaping our 'epigenomics'), through the organisms that cohabit with us (our 'microbiome') to atmospheric pollution in our environment (an example of the so-called 'exposome'). In the case of many common chronic degenerative diseases, our individual genome may therefore have limited impact: our nutrition, exercise patterns and many aspects of our environment, from the womb onwards, can be as important.

So I believe our definition of PM should take account of all the 'omic' measures relevant to a patient's disease and the factors affecting its course. We also should include in PM not just these 'omic' input measures but also the outputs that they guide, in terms of matching the intervention to the individual. For example, prognostic tests based on metabolomics and proteomics will result in recommendations to a presymptomatic individual at risk of metabolic syndrome to change their diet or exercise regimen and thereby prevent the development of diagnosable diabetes. Such 'precision' lifestyle interventions may also be guided by measures such as heart age and telomere length.

In addition to prescribing PMs, we will also deploy personalized digital tools to prompt measure and monitor the effects of treatment via a wearable or smartphone. Apps to support lifestyle management and therapy adherence will be customized to reflect individual health status and psychological makeup. They will also enable patients to join communities or compete with comparable individuals, to sustain their interest and commitment. 
Paradoxically, we may also include in our broad definition of PM the application of machine learning to major bodies of medical and lifestyle health data so that we may more precisely identify what treatments and lifestyle choices work best for individuals with different profiles.

\section{How will science shape PM?}

The primary driving force behind PM is clearly the science. Although genomics has so far had the highest profile, we will see other rapidly advancing 'omic' methodologies, like gut microbiomics and proteomic and metabolomic signatures in the blood contribute just as powerfully, reflecting as they do individual diet, environment and past treatments.

However biomedical science advances need to be integrated into clinical practice, both at the clinical trial stage and in routine care. Many companies now routinely profile patients 'omically' prior to admitting them to clinical trials, either as inclusion criteria or as a basis for retrospective analysis when responses vary. Building on the UK's 100,000 genomes project, the UK's Chief Medical Officer has just published a call for genomic medicine to be firmly embedded in the National Health Service [1].

Greater precision will also come via increasingly sophisticated medical images, analyzed by advanced algorithms. The application of MRI to diagnosis and treatment choice in prostate cancer is a particularly telling example, replacing random biopsies with an accurate picture of the pattern of lesions in an individual's prostate [2].

Finally, what could be more precise than the use of gene editing to remove or replace deleterious gene mutations, now demonstrated on human embryonic cells? [3].

\section{What nonscientific forces will influence its arrival?}

Nonscientific forces will also shape the future evolution of PM and its impact on healthcare. Societal attitudes to the collection and analysis of health data are fickle: while patients are excited by the results of research and generally support data sharing to advance it, the general public demands reassurance on data confidentiality. This is particularly true for our very personal genomic data, to safeguard it against inappropriate sharing with employers, insurers and even family members. Media attitudes to this complex area are also unpredictable.

Political initiatives can be useful in spurring the field. For the UK's 100,000 Genomes project, focused on cancer, rare and infectious disease, 'PM' also stood for Prime Minister, whose sponsorship launched the programme [4]. Likewise President Obama's Precision Medicine Initiative (PMI), that promises to bring genomic medicine to all strata of US society [5].

\section{Where will PM arrive first \& where later?}

Our PM future will undoubtedly be 'unevenly distributed' for some time to come, both in terms of diseases and geography.

Genomic and proteomic profiling of cancer is now quite well established in major cancer centers, although we will see many new discoveries as we apply whole genome sequencing to more and more tumors. Likewise the early use of exome sequencing and whole genome sequencing to rare diseases is becoming standard in many places. It can sharply curtail the typical 'diagnostic odyssey' (from one specialist to another) for many rare disease patients, providing an unambiguous diagnosis, sometimes leading to an immediate treatment, but at least an end to uncertainty.

Arrival in other disease areas will be more protracted. In diabetes, monogenic forms of the disease are already addressed by genomically guided PM; Type 1 diabetes seems likely to be next, although patients seem to vary widely in the number and nature of the dozens of genes now known to be involved in causation of the disease [6]. Type 2 is likely to be last, with a plethora of potential contributing factors, with the patient's genomic predisposition triggered by epigenomic or other factors of the metabolic syndrome(s) that seems to be at the center of causation for so many age-related chronic diseases.

However some chronic diseases are already seeing the impact of PM. In asthma and chronic obstructive pulmonary disease, doctors are distinguishing patients with eosinophilic airway inflammation requiring more aggressive treatment and monitoring [7]; in heart failure, a panel of proteomic tests promises a similar distinction [8].

While the cost of PM tests will continue to drop, the more sophisticated will clearly not be affordable everywhere on the planet. Many countries are investing to some extent in genomics, but the less affluent economies will probably need to wait until the findings are converted into inexpensive gene chips and blood tests before PM can make its impact in their health systems. 


\section{Which stakeholders will need to act to make PM a reality?}

The emergence of PM in practice will depend on the interplay between several stakeholders. Patients will engage to different extents, but the most alert and informed will use their test results to demand what is best for them, especially in life-threatening areas such as cancer. For doctors, PM poses major medical education and clinical practice challenges, as they face such patients and as every week brings new PM science. Industry is already building PM into their research and development strategies and will increasingly be focused on finding the right patients for their therapies, especially if payers reward them for outcomes achieved, not just the sale of their products.

Payers themselves are probably the most skeptical at this point, fearing, with some justification, ever-escalating prices. But they too will embrace the new era if they are offered solutions that combine targeting diagnostics, targeted therapies and tracking digital tools. The result will be fewer occasions on which they pay for ineffective treatment and indeed the potential for fewer treatment episodes, as a result of more targeted prevention.

\section{What will be the ultimate impact on healthcare?}

It is hard to overestimate the impact of PM on the future of healthcare. It will rescue the unsustainably low productivity of the biomedical innovation process, by improving its cost, time and attrition rate [9]. Although the more targeted treatments that emerge will be more expensive, they will increasingly banish the cost and complexities of 'trial and error' medicine. However, to fully harvest this potential, new generations of new precision diagnostic, prognostic, therapeutic and digital products will need to be matched with new thinking and new practice on the part of all the participants in the healthcare system.

\section{Financial \& competing interests disclosure}

RW Barker is a director of New Medicine Partners and a nonexecutive director of Celgene Corporation and the Image Analysis Group. The work of CASMI is supported by the Wellcome Trust, Cancer Research UK and the Innovative Medicines Initiative. The author has no other relevant affiliations or financial involvement with any organization or entity with a financial interest in or financial conflict with the subject matter or materials discussed in the manuscript apart from those disclosed.

No writing assistance was utilized in the production of this manuscript.

\section{References}

1 Davies PDS. Chief Medical Officer Independent Report 2016: Generation Genome. Department of Health, London, UK (2017). www.gov.uk/.../chief-medical-officer-annual-report-2016-generation-genome

2 Dickinson L, Ahmed HU, Allen C et al. Magnetic resonance imaging for the detection, localization and characterization of prostate cancer: recommendations from a European consensus meeting. Eur. Urol. 59(4), 477-494 (2011).

3 Ma H. Correction of a pathogenic gene mutation in human embryos. Nature 548(7668), 413-419 (2017).

4 Genomics England. The 100,000 Genomes Project (2012). www.genomicsengland.co.uk/the-100000-genomes-project/

5 Obama White House. Precision Medicine Initiative (2015). https://obamawhitehouse.archives.gov/node/333101

6 Hattersley A, Ellard S. Genetic Testing for Neonatal Diabetes (2004). www.diabetesgenes.org/content/genetic-testing-diabetes-presenting-infancy

$7 \quad$ Kulkarni NS, Hollins F, Sutcliffe A, Saunders R, Shah S et al. Eosinophil protein in airway macrophages: a novel biomarker of eosinophilic inflammation in patients with asthma. J. Allergy Clin. Immunol. 126(1), 61.e3-69.e3 (2010).

8 Ganz P, Heidecker B, Hveem K et al. Stratification of cardiovascular risk using an unbiased proteomic approach. JAMA 315(23), 2532-2541 (2016).

9 Barker RW. Bioscience - Lost In Translation? How Precision Medicine Closes The Innovation Gap. Oxford University Press, Oxford, UK (2016). 
